\title{
Modelling and energy management of a flywheel storage system for peak shaving applications
}

\author{
Lysandros Tziovani, Lenos Hadjidemetriou, Charalampos Charalampous, Stelios Timotheou, Elias Kyriakides \\ KIOS Research and Innovation Center of Excellence and Department of Electrical and Computer Engineering \\ University of Cyprus \\ Nicosia, Cyprus \\ \{ltziov01, lhadji02, cchara63, stimo, elias\}@ucy.ac.cy
}

\begin{abstract}
Peak shaving applications provided by energy storage systems are sustainable solutions for enhancing the existing capacity of distribution feeders and transformers in order to maintain their safe and reliable operation under an increased penetration of renewable energy sources and load demand growth. This work investigates the integration of a flywheel energy storage system installed in a feeder of a distribution network to provide peak shaving services. An empirical model is defined to determine the energy losses of a prototype flywheel system using an experimental setup. Furthermore, a multiobjective optimization scheme is proposed to minimize the flywheel energy losses along with the violated peak power of the feeder. Three different objective functions for applying peak shaving are presented and their efficiency is investigated in the simulation results. Finally, the impact of the flywheel energy losses on the peak shaving application of the distribution feeder is examined using a prototype and a commercial-grade flywheel energy storage system.
\end{abstract}

Index Terms-Distribution feeders, flywheel energy storage system, multi-objective optimization, peak shaving.

\section{INTRODUCTION}

The variable and intermittent generation of Photovoltaics (PVs) into the distribution grid along with the non-coincident peaks of load demand can result in the distribution feeders operating outside of their safe limits, especially in distribution grids with extensive integration of PVs and electric vehicle charging stations. Therefore, peak shaving applications provided by energy storage systems (ESS) are vital in maintaining the safe and reliable operation of distribution feeders [1]. ESSs enhance the existing capacity of distribution feeders to accommodate load and renewable energy generation growth in order to avoid any violations of the maximum power limit of the feeder line conductor or associated transformers [2]. Peak shaving applications are investigated in [3]-[5] for planning purposes, to examine the location, sizing and the cost-benefit of the ESSs in distribution feeders. Optimization methods to provide peak shaving services are proposed in [6]-[9]. The methods proposed in [6]-[7] aim to reduce the

This work is supported in part by the European Regional Development Fund and the Republic of Cyprus through the Research and Innovation Foundation (Project: INTEGRATED/0916/0035), and in part by European Union's Horizon 2020 research and innovation programme under grant agreement No 774407. It is also supported by the European Union's Horizon 2020 research and innovation programme under grant agreement No 739551 (KIOS CoE) and from the Government of the Republic of Cyprus through the Directorate General for European Programmes, Coordination and Development. peak load of the feeder for the next day by scheduling the charging/discharging of a battery energy storage system (BESS). A quadratic multi-objective optimization scheme is presented in [8] that minimizes both the square of the power drawn from the feeder to achieve peak shaving and the BESS life-cycle cost to increase the BESS lifetime.

This work investigates the integration of a Flywheel-based Energy Storage System (FESS) installed in a feeder of a distribution network to provide peak shaving services. Among the different types of ESSs, FESSs are characterized by full depth discharge capability, $85-90 \%$ efficiency rate, long lifetime (can reach 20 years or 100000 cycles), lower maintenance cost compared to chemical batteries and high charging-discharging abilities. These characteristics make FESSs suitable for applications that offer short-time power quality services and peakload regulation. Such short-time applications are proposed in [10]-[12] for power smoothing and surplus wind energy shifting in wind power plants. However, FESSs are not suitable for storing energy for long time periods because they suffer from high standby losses with an approximately $20 \%$ of selfdischarge per hour [13], [14]. Nevertheless, FESSs can be utilized to shave the violated peaks in a distribution feeder, if they are charged shortly before violations to provide the energy associated with the violated peaks. Towards this direction, this work proposes a multi-objective optimization scheme to minimize both the violated power of the feeder and the FESS energy losses. Despite FESSs having higher losses compared to BESSs, FESSs have longer lifetime and lower maintenance cost, which enhance their sustainability in such applications.

This work has two main novelties. The first is the empirical determination of the energy losses of a prototype FESS during its different operation modes by performing a number of experiments in our laboratory facilities. An investigation is performed to identify how the energy losses vary according to the charging-discharging rate and the state-of-charge of the FESS. The result is a piecewise linear model that provides the power losses of the FESS as a function of the state-of-charge and the charging-discharging rate. Furthermore, the experimental investigation identifies the variation of the maximum charging-discharging power of the FESS according to its stateof-charge, and the result is a piecewise linear function that approximates the nonlinear increment of the FESS maximum limits as the state-of-charge increases. 
The second main novelty of the paper is the utilization of the empirical model on energy losses for the formulation of a multi-objective moving-horizon optimization scheme for the minimization of the violated peaks and the FESS losses. Specifically, the proposed optimization scheme incorporates logical constraints in relation to the derived model on energy losses and the FESS maximum power, resulting in a MixedInteger Linear Program (MILP). Within the proposed scheme, an investigation is performed to determine the effectiveness of three different objective functions on the peak shaving capabilities of the FESS. Moreover, the impact of FESS losses in peak shaving applications is investigated using a prototype and a commercial-grade FESS [13]. The proposed scheme allows increased hosting capacity for PV installations and load growth by maximizing the utilization of the existing capacity of distribution feeders.

The rest of this paper is organized as follows. In Section II the FESS empirical model is described, followed by the problem formulation in Section III. Results are shown in Section IV and conclusions are given in Section V.

\section{FESS EMPIRICAL MODEL OF ENERGy LOSSES}

This section describes the derivation of an empirical model of the energy losses of a real FESS using the facilities of our power systems laboratory presented in Figure 1. The FESS consists of two parallel connected flywheels, with $6 \mathrm{~kW}$ total rated power and a usable capacity of $1.85 \mathrm{kWh}$. It must be noted that the FESS installed in our laboratory is an early prototype that operates under non-optimal vacuum conditions leading to high friction power losses. Nevertheless, the proposed analysis can be applied for empirically determining the energy losses of any commercial FESS operating in optimal vacuum conditions.

The variation of the power losses according to the charging rate, and the State-of-Charge (SoC) of the FESS are presented in Figure 2. As can be seen, the FESS power losses become higher as the charging power and the SoC increase. The power losses are derived from the difference between the measured absorbed energy from the grid and the measured stored energy in the FESS for a specific increase of the SoC

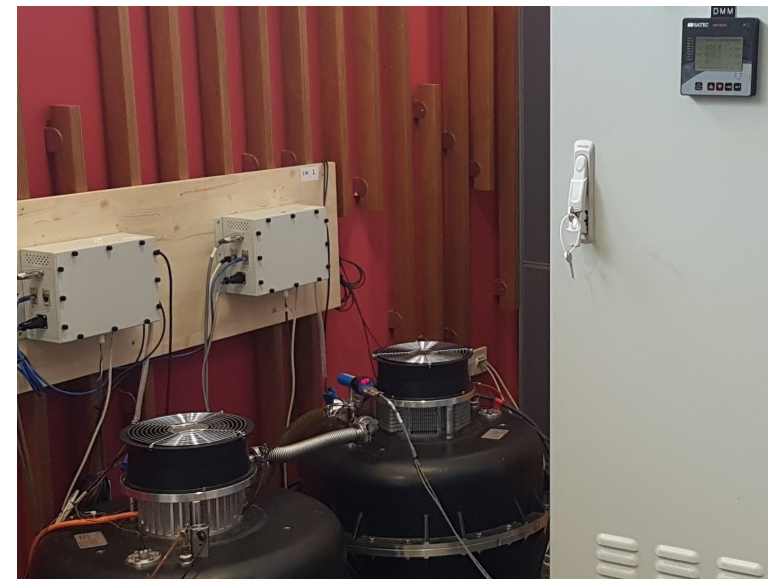

Fig. 1. FESS testbed facilities.

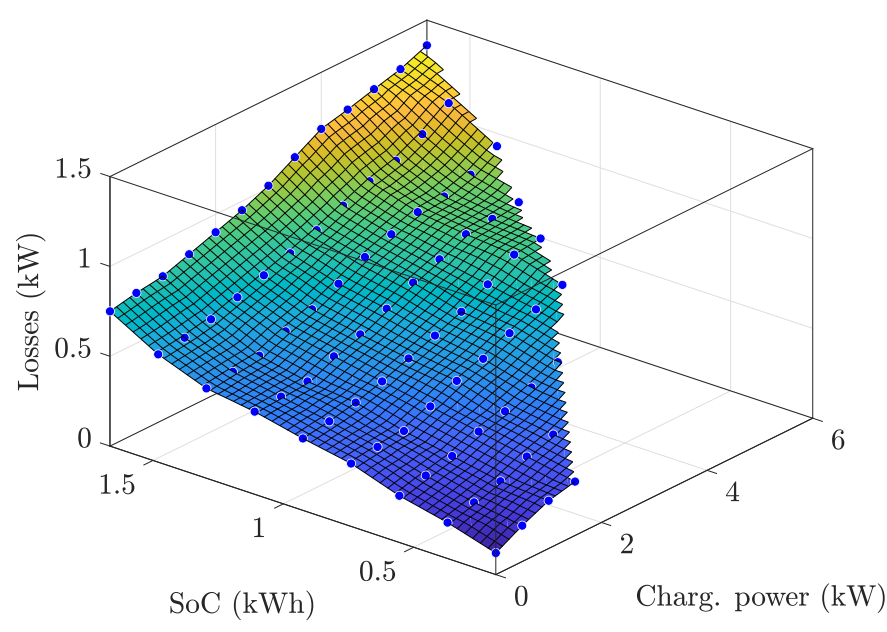

Fig. 2. The FESS power losses for varying state-of-charge and charging power. Note that the dots indicate sample points.

using a constant charging power. For example, the power losses at $50 \%$ SoC and $2 \mathrm{~kW}$ charging power are derived by the difference between the energy drawn from the grid and the stored energy within the FESS for a SoC increase from $45 \%$ to $55 \%$ using a constant charging power of $2 \mathrm{~kW}$. The same approach is used for the discharging mode. In addition, note that the maximum charging/discharging power in the FESS is depended on its SoC so that there is a higher maximum power limit for higher SoC values. Figure 3 indicates that the maximum charging/discharging power as a function of the SoC is a monotonically increasing concave function.

In this work, the FESS power losses are determined from linear regression using the $\mathrm{SoC}(\mathrm{kWh})$ and the charging or discharging rate $(\mathrm{kW})$ as independent variables and the power losses $(\mathrm{kW})$ as the dependent variable. Two different linear models are derived one for the charging mode and one for the discharging mode. Table I presents the parameters of the two derived models along with two coefficients to determine the goodness of fit. The Adjusted Coefficient of Determination (Adjusted $\mathrm{R}^{2}$ ) falls very close to 1 (Adjusted $\mathrm{R}^{2}>0.95$ ) in both cases; this indicates that the derived models explain more than $95 \%$ of the variance in the power losses. The goodness of fit is also indicated by the small Root Mean Squared Error (RMSE) values which are less than $0.1 \mathrm{~kW}$ in both cases. It should be noted that the obtained modelling error incorporates all measurement inaccuracies during the experimental investigation. Furthermore, the monotonically increasing concave function of the maximum charging/discharging power as a function of the $\mathrm{SoC}$ is approximated as a piecewise linear function with 10 linear segments, as illustrated in Figure

TABLE I

LINEAR REGRESSION - FESS POWER LOSSES (KW)

\begin{tabular}{c|c}
\hline Charging Mode & Discharging Mode \\
\hline$L^{c}=0.03+0.11 P^{c}+0.37 C^{f}$ & $L^{d}=0.005+0.22 P^{d}+0.395 C^{f}$ \\
Adjusted $\mathrm{R}^{2}=0.975$ & Adjusted $\mathrm{R}^{2}=0.967$ \\
RMSE $=0.045$ & RMSE $=0.086$ \\
\hline
\end{tabular}




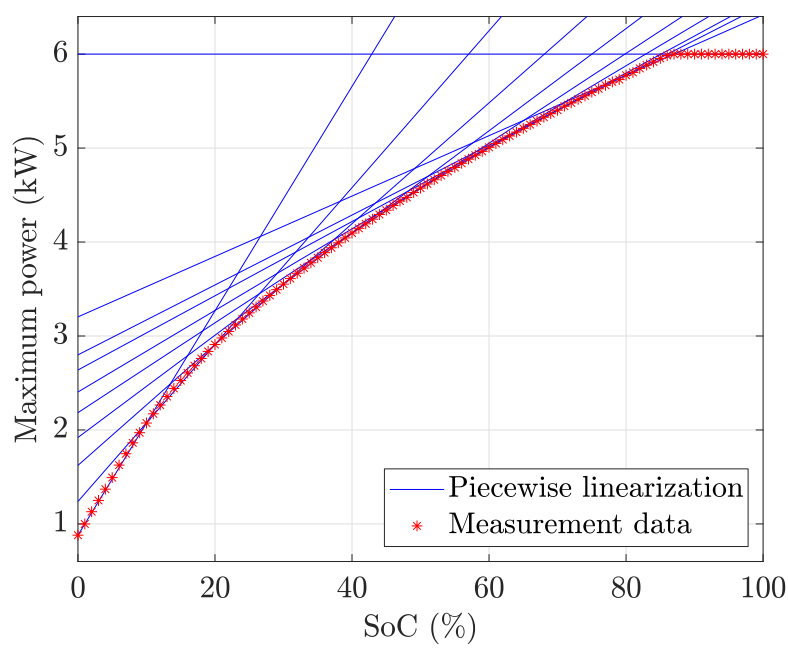

Fig. 3. The maximum charging and discharging power of the FESS as a function of the SoC obtained from 100 experimental samples. The ten blue solid lines construct a piecewise linear approximation of the concave function produced from the samples.

3. The derived linear functions of the FESS power losses along with the piecewise approximation are incorporated in the formulation of the proposed optimization scheme described in Section III.

\section{PROBLEM FORMULATION}

This section proposes a mathematical formulation to optimize the operation of the FESS in a way that provides a peak shaving service to a distribution feeder. Next, three multiobjective functions and the constraints of the problem are described in detail.

\section{A. Multi-objective Functions}

The objective of this work is to operate the feeder under its maximum power limit and to minimize the FESS energy losses over the entire time horizon. Towards this direction, three multi-objective functions are considered that minimize the FESS losses and penalize any excess power above the maximum power limit of the feeder.

Let $P_{t}^{l}$ and $x_{t}$ denote the FESS power losses in $\mathrm{kW}$ and the excess power above the nominal maximum power at time $t$, respectively. Let also $\mathcal{T}=\{1, \ldots, T\}$ denote the considered time horizon and $\Delta T$ denote the time-slot length in hours, such that a value of $\Delta T=1 / 4$ indicates 15 -minute timeslots. A large valued weight $M$ is used in all three objectives such that the peak shaving term is the dominating one in all functions. The three multi-objective functions are:

1) Linear multi-objective function: In (1a) the first term indicates the total FESS energy losses while the second term penalizes linearly the excess power. This penalty acts as an $l_{1}$-norm regularization term that aims to induce sparsity in $x_{t}$, $\forall t \in \mathcal{T}$.

$$
\min \sum_{t=1}^{T}\left(P_{t}^{l} \Delta T+M x_{t}\right)
$$

2) Quadratic multi-objective function: In addition to the total FESS energy losses, this objective penalizes the square of the excess power. This penalty acts as an $l_{2}$-norm regularization term that aims to reduce the magnitude of $x_{t}, \forall t \in \mathcal{T}$.

$$
\min \sum_{t=1}^{T}\left(P_{t}^{l} \Delta T+M x_{t}^{2}\right)
$$

3) Minimax multi-objective function: In addition to the total FESS energy losses, this objective penalizes the maximum of the excess power. Objective (1c) can be reformulated into a linear objective with an additional set of constraints, as shown in $(1 \mathrm{~d})$.

$$
\begin{gathered}
\min \sum_{t=1}^{T}\left(P_{t}^{l} \Delta T\right)+M \max _{t}\left(x_{t}\right) \\
\min \quad \sum_{t=1}^{T}\left(P_{t}^{l} \Delta T\right)+M z \\
\text { s.t. } x_{t} \leq z, \quad \forall t \in \mathcal{T}
\end{gathered}
$$

\section{B. Constraints}

1) Peak shaving constraints: The constraints related to the peak shaving are the feeder power balance (2a), and the soft constraint for the feeder maximum power limit (2b)

$$
\begin{aligned}
P_{t}^{F}+P_{t}^{d} & =\hat{P}_{t}^{L}+P_{t}^{c}, \quad P_{t}^{c} \perp P_{t}^{d} \quad \forall t \in \mathcal{T}, \\
0 & \leq P_{t}^{F} \leq \bar{P}^{F}+x_{t}, \quad \forall t \in \mathcal{T},
\end{aligned}
$$

where $\hat{P}_{t}^{L}$ is the predicted power demand of the feeder in $\mathrm{kW}, P_{t}^{c}$ is the FESS charging power in $\mathrm{kW}, P_{t}^{d}$ is the FESS discharging power in $\mathrm{kW}, P_{t}^{F}$ is the power of the feeder in $\mathrm{kW}$ and $\bar{P}^{F}$ is the maximum power limit of the feeder in $\mathrm{kW}$. Note that variables $P_{t}^{c}$ and $P_{t}^{d}$ are complementary to each other which means that only one of the two variables can be non-zero.

2) FESS state of charge: In (2c), the stored energy (kWh) in the FESS for the next time interval is equal to the stored energy $\left(C_{t}^{f}\right)$ in the current interval plus the energy imported to the FESS due to the charging power, and minus the energy exported from the FESS due to the discharging power and the FESS energy losses. Also, in (2d) the stored energy in the FESS must be less than its maximum limit $\left(\bar{C}_{t}^{f}\right)$. Note that $I^{c}$ is the initial energy stored in the FESS.

$$
\begin{gathered}
C_{t+1}^{f}=C_{t}^{f}+\Delta T\left(P_{t}^{c}-P_{t}^{d}-P_{t}^{l}\right), \quad \forall t \in \mathcal{T} \\
C_{0}^{f}=I^{c}, \quad 0 \leq C_{t}^{f} \leq \bar{C}_{t}^{f}, \quad \forall t \in \mathcal{T}
\end{gathered}
$$

3) FESS maximum charging and discharging power: To derive constraints on the maximum charging and discharging power, the piecewise linear functions derived in Figure 3 are exploited. Let the $i$-th linear segment be given by $\alpha_{i}+\beta_{i} C_{t}^{f}$, $i \in \mathcal{N}=\{1, \ldots, N\}$. Then, constraints $(2 \mathrm{e})-(2 \mathrm{~g})$ provide the maximum values for $P_{t}^{c}$ and $P_{t}^{d}$ for a given SoC; $\bar{P}^{c}$ and $\bar{P}^{d}$ are the upper bounds of these variables.

$$
\begin{aligned}
P_{t}^{c} \leq \alpha_{i}+\beta_{i} C_{t}^{f}, & \forall i \in \mathcal{N}, \forall t \in \mathcal{T} \\
P_{t}^{d} \leq \alpha_{i}+\beta_{i} C_{t}^{f}, & \forall i \in \mathcal{N}, \forall t \in \mathcal{T} \\
0 & \leq P_{t}^{c} \leq \bar{P}^{c}, \quad 0 \leq P_{t}^{d} \leq \bar{P}^{d}, \forall t \in \mathcal{T}
\end{aligned}
$$


TABLE II

FESS POWER LOSSES

\begin{tabular}{c|cc}
\hline ID & FESS Logical Constraints \\
\hline FC1 & $P_{t}^{l}=P_{t}^{l, d}\left(1-b_{t}\right)+P_{t}^{l, c}\left(b_{t}\right), \quad b_{t} \in\{0,1\}, \quad \forall t \in \mathcal{T}$ \\
FC2 & $P_{t}^{l, d}=\hat{A}^{d} b_{t}^{d}+\hat{B}^{d} P_{t}^{d}+\hat{C}^{d} C_{t}^{f}, \quad b_{t}^{d} \in\{0,1\}, \quad \forall t \in \mathcal{T}$ \\
FC3 & $P_{t}^{l, c}=\hat{A}^{c} b_{t}^{c}+\hat{B}^{c} P_{t}^{c}+\hat{C}^{c} C_{t}^{f}, \quad b_{t}^{c} \in\{0,1\}, \quad \forall t \in \mathcal{T}$ \\
FC4 & $P_{t}^{c} \geq \hat{\epsilon} \Longleftrightarrow b_{t}=1, \quad \forall t \in \mathcal{T}$ \\
FC5 & $C_{t}^{f} \geq \hat{A}^{d} \Delta T \Rightarrow b_{t}^{d}=1, \quad C_{t}^{f} \geq \hat{A}^{c} \Delta T \Rightarrow b_{t}^{c}=1, \quad \forall t \in \mathcal{T}$ \\
\hline
\end{tabular}

TABLE III

EQUIVALENT MILP EXPRESSIONS

\begin{tabular}{c|c|c}
\hline ID & Constraints & MILP Expressions \\
\hline MC1 & $y=x \delta, \quad \delta \in\{0,1\}$ & $L \delta \leq y \leq U \delta, \quad L \leq x \leq U$, \\
MC2 & $x \geq c \Rightarrow \delta=1$ & $L(1-\delta) \leq x-y \leq U(1-\delta)$ \\
& & $x-(U+\epsilon) \delta \leq c-\epsilon$, \\
MC3 & $x \geq c \Longleftrightarrow \delta=1$ & $L(1-\delta) \leq x-c \leq(U+\epsilon) \delta-\epsilon$, \\
& & $U=\max (0, \overline{x-c})$ \\
& & $L=\min (-\epsilon, \underline{x-c})$ \\
\hline
\end{tabular}

4) FESS power losses: Incorporating the derived power losses models, presented in Table I, into the problem formulation is challenging because the model to be selected depends on the charging mode. To achieve this, we introduce the logical constraints FC1 to FC5 in Table II. FC2 and FC3 indicate the charging and discharging power losses, $P_{t}^{l, c}$ and $P_{t}^{l, d}$ respectively, where the parameters $\hat{A}^{d}, \hat{B}^{d}, \hat{C}^{d}, \hat{A}^{c}, \hat{B}^{c}$ and $\hat{C}^{c}$ are defined by the corresponding estimated coefficients in Table I. Constraints FC5 ensure that parameters $\hat{A}^{d}$ and $\hat{A}^{c}$ are considered in FC2 and FC3 only when there is stored energy in the FESS. Constraints FC4 ensure that when $P_{t}^{c}>0$ then $b_{t}=1$. Then, Constraints FC1 and FC3 ensure that the charging power loss model is selected. In a similar fashion the discharging loss model is selected when $P_{t}^{d}>0$.

The logical constraints FC1-FC5 in Table II are included in the formulation using the equivalent MILP transformations MC1-MC3 presented in Table III. Therefore, constraints (2h)(2k) represent logical constraint FC1 using the MILP transformation in $\mathrm{MC1}$, where $Y_{t}^{d}$ and $Y_{t}^{c}$ are equal to the products $P_{t}^{l, d} b_{t}$ and $P_{t}^{l, c} b_{t}$, respectively. $U$ is an upper bound of $Y_{t}^{d}$ and $Y_{t}^{c}$ and is set to $\bar{C}_{t}^{f} / \Delta T$ (where $Y_{t}^{c} \Delta T \leq \bar{C}_{t}^{f}$ ). Similarly, constraint (2l) represents FC4 using the MILP transformation in MC3. Note that $\epsilon$ and $\hat{\epsilon}$ are infinitesimally small values where $\epsilon<\hat{\epsilon}$. Constraints (2m) and (2n) represent FC2 and FC3, while constraints (2o) and (2p) represent FC5 using the MILP transformation in MC2.

$$
\begin{gathered}
P_{t}^{l}=P_{t}^{l, d}-Y_{t}^{d}+Y_{t}^{c}, \quad \forall t \in \mathcal{T} \\
0 \leq Y_{t}^{d} \leq b_{t} U, \quad 0 \leq Y_{t}^{c} \leq b_{t} U, \quad \forall t \in \mathcal{T} \\
0 \leq P_{t}^{l, d}-Y_{t}^{d} \leq\left(1-b_{t}\right) U, \quad \forall t \in \mathcal{T} \\
0 \leq P_{t}^{l, c}-Y_{t}^{c} \leq\left(1-b_{t}\right) U, \quad \forall t \in \mathcal{T} \\
-\bar{P}_{t}^{c}\left(1-b_{t}\right) \leq P_{t}^{c}-\hat{\epsilon} \leq\left(\bar{P}_{t}^{c}+\epsilon\right) b_{t}-\epsilon, \quad \forall t \in \mathcal{T}
\end{gathered}
$$

(a)

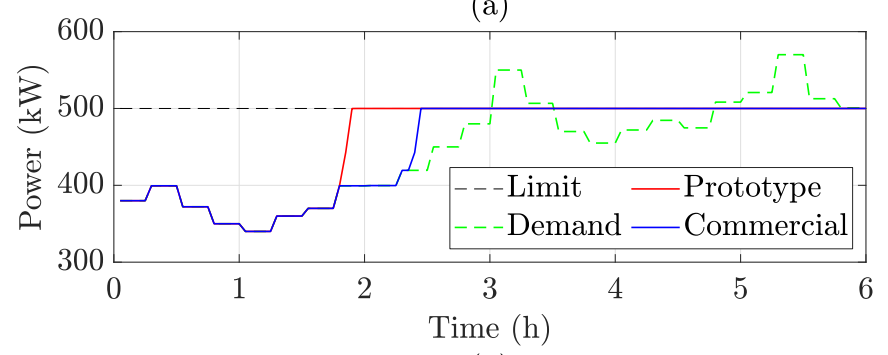

(b)

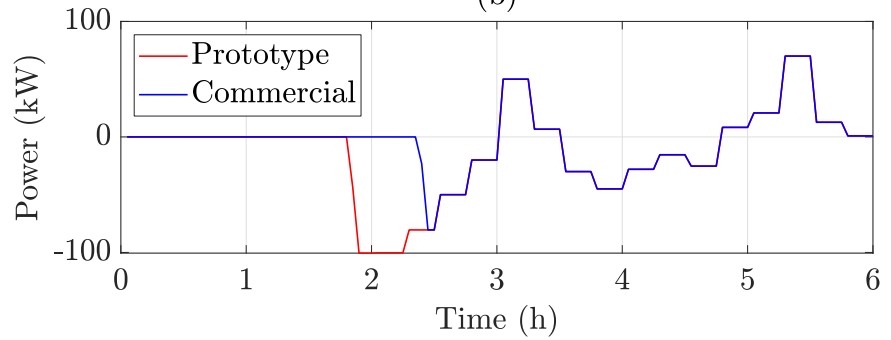

(c)

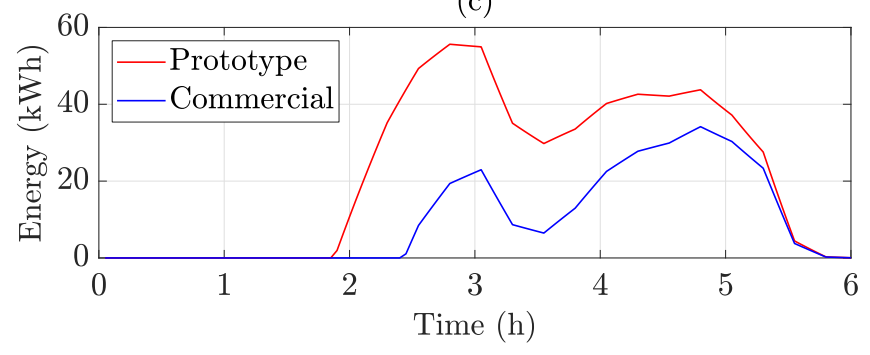

Fig. 4. (a) Feeder operation using the prototype and a commercial FESS. (b) Discharging and charging power. (c) State-of-Charge.

$$
\begin{array}{cc}
P_{t}^{l, d}=\hat{A}^{d} b_{t}^{d}+\hat{B}^{d} P_{t}^{d}+\hat{C}^{d} C_{t}^{f}, & \forall t \in \mathcal{T} \\
P_{t}^{l, c}=\hat{A}^{c} b_{t}^{c}+\hat{B}^{c} P_{t}^{c}+\hat{C}^{c} C_{t}^{f}, & \forall t \in \mathcal{T} \\
C_{t}^{f}-\left(\bar{C}_{t}^{f}+\epsilon\right) b_{t}^{d} \leq \hat{A}^{d} \Delta T-\epsilon, & \forall t \in \mathcal{T} \\
C_{t}^{f}-\left(\bar{C}_{t}^{f}+\epsilon\right) b_{t}^{c} \leq \hat{A}^{c} \Delta T-\epsilon, & \forall t \in \mathcal{T}
\end{array}
$$

\section{Simulation Results}

For the simulation validation of our work we have considered a 100-times scale-up version of the prototype FESS described in Section II resulting in a FESS with $185 \mathrm{kWh}$ and $600 \mathrm{~kW}$ power. The FESS is integrated with a distribution feeder with maximum limit $500 \mathrm{~kW}$. Note that the proposed peak shaving application is used for operational purposes, while planning purposes are out of the scope of this work.

For the simulation we consider a horizon of 6 hours separated into 3 minute time-intervals because the high FESS standby losses do not allow energy storage for a long time. The mathematical formulation is coded in MATLAB and the optimization solver Gurobi is used to solve the MILP problem. The results of the peak shaving application on the distribution feeder using the proposed optimization scheme are presented in Figure 4. Figure 4(a) shows the predicted load demand of the feeder, the operation of the feeder using the prototype FESS with the estimated losses coefficients, and the feeder operation using a commercial FESS. Note that an efficiency of $85 \%$ and $20 \%$ standby losses per hour are considered for 


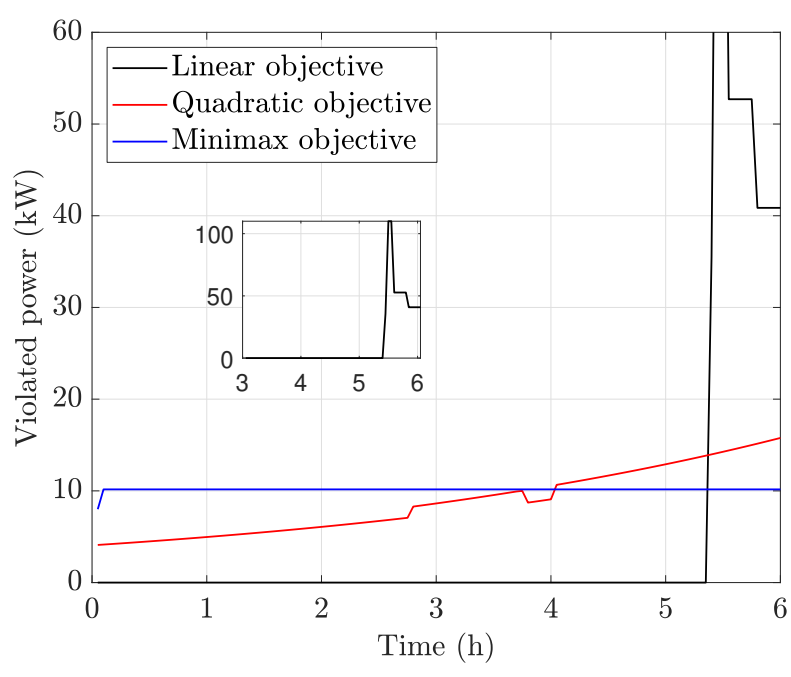

Fig. 5. Power violations using the three objective functions.

the commercial FESS [13]; thus, the coefficients of the power losses are set to $\hat{A}^{d}=\hat{A}^{c}=0, \hat{B}^{d}=\hat{B}^{c}=0.075$, and $\hat{C}^{d}=\hat{C}^{c}=0.2$. In addition, parameter $M$ is set equal to 1000. The results indicate the effectiveness of the proposed scheme to shave the peaks above the maximum limit of 500 $\mathrm{kW}$ for both cases. However, the prototype FESS is charged for a longer period (see Figure 4(b)) and stores more energy (see Figure 4(c)) compared to the commercial FESS which is due to its very high friction power losses. As a result, the energy losses of the prototype system are $73.5 \mathrm{kWh}$ which are extremely high compared to the $20.3 \mathrm{kWh}$ of the commercial system. Note that the results of the three objective functions in (1a)-(1c) are the same for this scenario, since both systems manage to shave the peaks and there is no violated power. The investigation indicates that considering an adequate model of FESS energy losses is important for such peak shaving applications. Thus, the empirical losses modeling approach used in this work can be utilized to determine the losses of any prototype or commercial FESS, enabling an accurate scheduling of FESS in such energy management applications.

The effectiveness of the three objective functions (1a)-(1c) is presented in Figure 5, when the FESS is not able to shave the power peaks and violations do occur. Towards this direction, it is assumed that the maximum limit of the feeder is $460 \mathrm{~kW}$, and the case of the commercial FESS is investigated, while the load demand remains the same with the one presented in 4(a). The linear objective function manages to eliminate the power violations for the most of the period, but extremely high power violations of $110 \mathrm{~kW}$ are presented for the rest of the period. However, these high power violations can damage the feeder, and as a result the linear objective function is not suitable to minimize the power violations of the feeder. The quadratic objective function manages to reduce the violations under the $16 \mathrm{~kW}$ ( $3.5 \%$ of overloading conditions), whereas the minimax objective function finds the minimum power violation of 10.1 $\mathrm{kW}$ ( $2.2 \%$ of overloading conditions). The energy losses of the minimax objective are $134 \mathrm{kWh}$ which are higher than the $126 \mathrm{kWh}$ and $109 \mathrm{kWh}$ of the quadratic and the linear objectives, however better protecting the feeder by minimizing the violated power peaks is more important than the reduction of the energy losses. Therefore, the minimax objective function performs better in the provision of peak shaving services to a distribution feeder compared to the rest objective functions.

\section{CONCLUSIONS}

In this work, a novel optimization scheme is proposed to provide peak shaving services to a distribution feeder with an integrated FESS. The energy losses of a real prototype FESS are empirically determined and the derived energy losses are incorporated in the optimization scheme. The effectiveness of three objective functions on the reduction of the peak power violations is also examined. Furthermore, this study evaluates how the empirical modelling of a real FESS is essential to reduce the uncertainties between real systems and simulation models which can lead to undesirable and unsafe operating states. Uncertainties associated with the predicted input data will be addressed in a future work by applying the proposed scheme in a model predictive control framework. Finally, future work will test the experimental verification of the proposed methodology.

\section{REFERENCES}

[1] B. P. Roberts et al., "The role of energy storage in development of smart grids," in Proc. of the IEEE, vol. 99, no. 6, pp. 1139-1144, Jun. 2011

[2] M. H. Pandya and M. V. Aware, "Enhancing the distribution feeder capacity through energy storage," in Proc. IEEE ICIT, Cape Town, South Africa, 2013, pp. 1739-1744.

[3] Y. Yang et al., "Cost-benefit study of dispersed battery storage to increase penetration of photovoltaic systems on distribution feeders," in Proc. IEEE PES General Meeting, National Harbor, MD, 2014, pp. 1-5.

[4] T. Zhang, A. E. Emanuel and J. A. Orr, "Distribution feeder upgrade deferral through use of energy storage systems," in Proc. IEEE PES General Meeting, Boston, MA, 2016, pp. 1-5.

[5] A. O'Connellet al., "Integrating photovoltaic and storage systems on distribution feeders," in CIRED - Open Access Proceedings Journal, vol. 2017, no. 1, pp. 1831-1835, Oct. 2017.

[6] D. Kodaira, W. Jung and S. Han, "Optimal Energy Storage System Operation for Peak Reduction in a Distribution Network Using a Prediction Interval," in IEEE Trans. Smart Grid, Oct. 2019.

[7] J. Tant, F. Geth, D. Six, P. Tant and J. Driesen, "Multiobjective battery storage to improve PV integration in residential distribution grids," IEEE Trans. Sustain. Energy, vol. 4, no. 1, pp. 182-191, Jan. 2013.

[8] A. Nagarajan and R. Ayyanar, "Design and Strategy for the Deployment of Energy Storage Systems in a Distribution Feeder With Penetration of Renewable Resources," in IEEE Trans. Sustain. Energy, vol. 6, no. 3, pp. 1085-1092, July 2015.

[9] L. Tziovani et al., "Grid friendly operation of a PV-storage system with profit maximization and reliability enhancement," in Proc. IEEE SEST, Porto, Portogul, 2019, pp. 1-6.

[10] F. Díaz-González et al., "Control of a Flywheel Energy Storage System for Power Smoothing in Wind Power Plants," in IEEE Trans. Energy Conversion, vol. 29, no. 1, pp. 204-214, March 2014

[11] F. Díaz-González et al., "Energy management of flywheel-based energy storage device for wind power smoothing," in Applied Energy, vol. 110, pp. 207-219, Oct. 2013.

[12] H. H. Abdeltawab et al., "Robust Energy Management of a Hybrid Wind and Flywheel Energy Storage System Considering Flywheel Power Losses Minimization and Grid-Code Constraints," in IEEE Trans. Industrial Electronics, vol. 63, no. 7, pp. 4242-4254, July 2016.

[13] F. Nadeem et al., "Comparative Review of Energy Storage Systems, Their Roles, and Impacts on Future Power Systems," in IEEE Access, vol. 7, pp. 4555-4585, 2019.

[14] M. Farhadi and O. Mohammed, "Energy Storage Technologies for HighPower Applications," in IEEE Trans. Industry Applications, vol. 52, no. 3, pp. 1953-1961, 2016. 